CHRISTIAN RITZI

(Bibliothek für Bildungsgeschichtliche Forschung des Deutschen Instituts für Internationale Pädagogische Forschung Berlin)

\title{
ZUR WIEDERENTDECKUNG EINER HANDSCHRIFTLICHEN CHRONIK DES GYMNASIUMS ZU THORN VON JULIUS EMIL WERNICKE*
}

Von Ende 1993 bis Anfang 1994 musste die Bibliothek für Bildungsgeschichtliche Forschung (BBF) aus ihren bis dahin genutzten Räumen am Berliner Alexanderplatz ausziehen. Von diesem Umzug waren auch drei mächtige, noch aus DDR-Zeiten stammende Stahlschränke betroffen, obwohl sie im neuen Bibliothekskonzept zunächst keine Funktion mehr hatten. Dass einer der Schränke verschlossen und der zugehörige Schlüssel nicht mehr auffindbar war, wurde deshalb nur beiläufig zur Kenntnis genommen, zumal ältere Bibliotheksmitarbeiter versicherten, dass der Schrank leer sei.

* Erweiterte Fassung eines Vortrags vom 11. Dezember 2009 beim Forum Toruniense in Toruń (Polen). Überarbeitete Version eines Aufsatzes in „Mitteilungsblatt des Förderkreises Bibliothek für Bildungsgeschichtliche Forschung e.V.“ 21, 1 (2010), erhältlich im Internet http://www.bbf.dipf.de/foerderkreis/2010/mb_2010_01.pdf (besucht am 7. Juni 2011), S. 29-42. 
Als die BBF Ende der 1990er Jahre begann, ältere Zeitschriften und Nachschlagewerke zu digitalisieren, entstand ein Bedarf für die dauerhafte Lagerung der in diesem Zusammenhang erstellten Mikrofilme. Dazu schien der bislang verwaiste Safe geeignet, zumal er einen zusätzlichen Brandschutz versprach. Daraufhin wurde ein Schlosser mit dem Aufbrechen beauftragt, was zu einem erstaunlichen Fund führte. Denn der Stahlschrank erwies sich keineswegs als leer, sondern enthielt $u$. a. eine umfangreiche Handschrift. Es handelt sich um eine Chronik des 1568 gegründeten Gymnasiums zu Thorn, die dessen Geschichte von den Anfängen bis zur Mitte des 19. Jahrhunderts dokumentiert ${ }^{1}$.

Glücklicherweise fand sich unter den der Handschrift lose beigefügten Dokumenten eine Notiz des Verfassers, wodurch sich auch der Entstehungszeitraum eingrenzen lässt. In dem Text heißt es:

Enthält die Geschichte des Gymnasiums und der Thorner Schu-

len seit dem Ursprunge der Stadt bis zur Gegenwart und ist zu-

${ }^{1}$ Der hier zur Verfügung stehende Platz erlaubt es nicht, näher auf die Bedeutung dieses berühmten Gymnasiums einzugehen, vgl. u.a. K.F.A. Brohm, Geschichte des Thornschen Gymnasiums, „Schulprogramm des Gymnasiums zu Thorn“, (1819), S. 3-18; K.F.A. Brohm, Erinnerung an Heinrich Stroband, „Schulprogramm des Gymnasiums zu Thorn“, (1824), S. 3-10; M. Curtze, Die Handschriften und seltenen alten Drucke der Gymnasialbibliothek zu Thorn, „Schulprogramm des Gymnasiums zu Thorn“, (1875), S. 1-40; L. Kühnast, Zum 300jährigen Jubiläum des Thorner Gymnasiums, „Pädagogisches Archiv: Monatsschrift für Erziehung, Unterricht und Wissenschaft", 10, 4 (1868), S. 274-280; P. Rudolf, Vierhundert Jahre Thorner Gymnasium, in: Thorn - Stadt und Land, hg. v. H.-E. Krüger, (1981), S. 79-95. Neuere Literatur wurde insbesondere von polnischen Historikern veröffentlicht, vgl. u.a. S. Salmonowicz, Jesuitenschule und Akademische Gymnasien in Königlich Preussen (16.-18. Jh.), in: ders., Polen im 17. und 18. Jahrhundert: Abhandlungen und Aufsätze, (1997), S. 39-53; ders., Das Thorner protestantische Gymnasium Academicum 1568-1793, „Beiträge zur Geschichte Westpreußens“, 14 (1995), S. 41-3. 
sammengestellt aus allen gefundenen Quellen, die sich über diesen Gegenstand und irgendwo haben auffinden lassen.

S. 1-501 nebst Beilagen

Ich habe zur Abfassung dieses Werkes 7 Jahre gebraucht.

Wernicke

Damit ist der Autor der Handschrift leicht zu identifizieren. Es handelt sich um Julius Emil Wernicke, der am 18. Februar 1824 im Thorner Gymnasium als Lehrer angestellt wurde und dort bis zum Schuljahr 1844/1845 unterrichtete ${ }^{2}$. Geboren am 16. August 1802 in Breslau, besuchte er das dortige Elisabetanische Gymnasium und studierte $\mathrm{ab} 1819$ an der Berliner Universität zunächst Theologie, später Philologie und Geschichte. Im Anschluss besuchte Wernicke das pädagogische Seminar für gelehrte Schulen in Breslau, wo er auch erste Lehrerstellen am Magdaleneum ${ }^{3}$ und im Fridricianum ${ }^{4}$ erhielt. Wenige Tage nach Dienstantritt am Thorner Gymnasium wurde er am 27. Februar 1824 promoviert. Seine Unterrichtsfächer waren während des gesamten Zeitraums Griechisch und Latein, zeitweise auch Religion und ab dem Schuljahr 1838/1839 zusätzlich Geschichte und Geographie. In eben diesem Jahr rückte er an die erste Stelle im Lehrerkollegium auf ${ }^{5}$ und ein Jahr später, 1840, wurde ihm der Professorentitel erteilt ${ }^{6}$.

1845 bat er um seine vorzeitige Pensionierung, der offensichtlich Konflikte in der Schule vorausgingen. In seinem handschriftlichen Lebenslauf findet sich hierzu nur eine vage Andeutung:

2 Seine Versetzung in den Ruhestand wurde im „Schulprogramm des Gymnasiums zu Thorn“, (1846), S. 13 mitgeteilt.

3 Gymnasium zu St. Maria Magdalena in Breslau.

4 Königliches Friedrichs-Gymnasium.

5 Vgl. „Schulprogramm des Gymnasiums zu Thorn“, (1839), S. 14.

6 Vgl. „Schulprogramm des Gymnasiums zu Thorn“, (1840), S. 14. 
„Verfolgungen mancherlei Art (und gerade in Thorn recht grell hervortretend), bestimmten ihn schon im Jahre 1845 seine Entlassung nachzusuchen, die ihm Anfang 1846 seit März mit Pension ertheilt wurde "7. Anschließend blieb Wernicke noch in Thorn, um dann nach Berlin umzuziehen, wo er 1866 gestorben ist.

In den 20 Jahren seiner Thorner Unterrichtstätigkeit verfasste er vier gelehrte Abhandlungen zu Schulprogrammen des Thorner Gymnasiums sowie weitere publizierte Schriften ${ }^{8}$. Den bei weitem größten Forschungsaufwand investierte Wernicke jedoch in die hier vorgestellte Schulchronik. Weshalb er eine abschließende redaktionelle Bearbeitung des Manuskripts unterließ und auf eine Drucklegung verzichtete, ist nicht eindeutig aufzuklären. Naheliegend wäre, dass ihn der angedeutete Konflikt demotivierte, vielleicht sogar die Nutzung des Schularchivs behinderte. Immerhin blieb die Handschrift erhalten und seine umfangreichen Vorarbeiten konnten von späteren Historiographen des Thorner Gymnasiums für eigene Publikationen zumindest zeitweise genutzt werden.

Die Durchsicht der Handschrift ergibt folgende Gliederung:

- Scholarchen-Kollegium des Thorner Gymnasiums von 1584-1736. S. 1-36. Fortsetzung S. $487 \mathrm{ff}$

- Abschrift des Fundationsprivilegiums der Universität Kulm. S. 37-38

- Geschichte des Thornschen Gymnasiums und der übrigen mit ihm verbundenen Lehr- und wissenschaftlichen Anstalten. S. $39-400$

7 Vgl. den von ihm selbst verfassten transkribierten Lebenslauf im Anhang sowie ergänzend A. Lehnerdt, Geschichte des Gymnasiums zu Thorn, in: Fest-Programm des Königl. Evangelischen Gymnasiums zu Thorn, (1868), S. 1-52, hier S. 19.

$8 \mathrm{Vgl}$. das von ihm selbst zusammengestellte Schriftenverzeichnis in seinem Lebenslauf im Anhang. 
- Fragmentarische Geschichte der Thornschen öffentlichen Unterrichts-Anstalten bis zur privilegierten Reformation 1577. S. 43-44

- Die Johannisschule. S. 44-54

- Die Jakobsschule oder ältere Neustädtische Schule. S. 54-66. Fortsetzung S. 437

- Geschichte des Thorner Gymnasiums. S. 66-400

- Nachträge. S. 400-484

- Etat des Gymnasiums. S. 400

- Legate und Stipendien für Studierende. S. 401-407

- Das alte Alumnat, ,Oeconomie' genannt. S. 407-414

- Die Gymnasialbibliothek. S. 414-426

- Die Gymnasialbuchdruckerei. S. 426-436

- Die Neustädtische Schule. S. 437-463. Fortsetzung von S. 66

- Das Kollegium oder die Schule der Jesuiten. S. 463-483

- Anhang

- Von einigen Schulen zur Zeit des Freistaates. S. 484-486.

- Scholarchen-Kollegium. S. 487-497. Fortsetzung von S. 36

- Scholarchen der Neustädtischen Schule. S. 498-500

- Von den Landschulen der Kämmerei Thorn. S. 501-512 (diese Seiten wurden später neu gezählt: S. 575-587).

Die von Wernicke verfasste Handschrift umfasst 512 Seiten, die von ihm selbst paginiert wurden. In der oben erwähnten Autorennotiz gibt er allerdings nur 501 Seiten an (S. 1-501 nebst Beilagen), so dass der Text im unteren Seitendrittel der Seite 501 sowie die Seiten 502 bis 512 nach Abfassung der Notiz hinzugefügt wurden. Die auf den Seiten 1-501 dargestellte Schulgeschichte erstreckt sich bis in das Jahr 1847, so dass Wernicke - ausgehend von der in der Autorennotiz angegebenen Bearbeitungszeit von sieben Jahren - etwa 1840 mit ihrer Abfassung begonnen haben 
dürfte, mithin in der Zeit, als er auch Geschichts- und Geographieunterricht erteilte.

Neben den Seiten 501 (unteres Drittel) bis 512 wurden auch zahlreiche Ergänzungen auf den Seitenrändern nach 1847 geschrieben. Ebenfalls zu einem späteren Zeitpunkt wurden von Wernicke gesammelte oder später von anderen beigetragene gedruckte und ungedruckte Dokumente in die Chronik eingeschoben, deren Zählung allerdings von anderer Hand erfolgte und in der Regel mit der vorhergehenden Seitenzahl der Chronik versehen wurde, ergänzt um einen Buchstaben (z.B. 226a, 268a). Weitere Dokumente wurden nach Seite 500 eingebunden und unter Fortführung der Wernickeschen Zählung mit anderer Hand paginiert. Die letzten der von Wernicke geschriebenen Seiten (S. 501-512) wurden an das Ende gebunden und mit neuer Zählung versehen (S. 575-587; die letzte Seite ist ohne neue Paginierung). Die beigefügten bzw. eingeschobenen Dokumente wurden allerdings nur zum Teil seitenweise paginiert, andere blatt- oder gar dokumentenweise. Dadurch erhöht sich die tatsächliche Seitenzahl gegenüber der mit Paginierung versehenen beträchtlich. Darüber hinaus gibt es noch einen Anhang mit zum Teil mehrseitigen und lose beigelegten Dokumenten, die überhaupt keine Zählung aufweisen.

Bis auf die lose beigelegten Dokumente, die in einer aufgeklebten Tasche auf der dritten Einbandseite verwahrt sind, wurden alle Blätter in einem etwa aus den 1960er Jahren stammenden Einband eingebunden. Mutmaßlich in diesem Zusammenhang wurden auch restauratorische Maßnahmen ergriffen wie etwa das Kaschieren einzelner Blätter.

Der vom Umfang her größte Teil der Handschrift führt von Jahr zu Jahr Veränderungen im Thorner Gymnasium auf. Jeweils neu hinzugekommene Rektoren und Lehrer werden vorgestellt, ihr Ausbildungsgang, ihre Familienverhältnisse und ihre Schriften. Berichte oder Dokumente zu besonderen Vorkommnissen 
in der Schule finden sich in den Beilagen, darunter Abschriften Wernickes, aber auch Originaldokumente, so etwa eine mit Wachssiegel versehene Urkunde aus dem Jahr 1557.

Schwierig ist die Rekonstruktion des Weges, den die Handschrift bis zu ihrem heutigen Standort in der BBF zurücklegte. Der einzige Besitzstempel in der Handschrift ermöglicht keine Zuordnung, da er unvollständig und ausgebleicht ist. Ein erster Hinweis auf einen Standort findet sich im „Fest-Programm zur Feier des 300jährigen Bestehens der Anstalt", in dem der damalige Rektor des Thorner Gymnasiums, Ludwig Moritz Albert Lehnerdt, einen Abriss zur Schulgeschichte publizierte9. Darin widmet er auch einen Abschnitt den Quellen und Hülfsmitteln, die ihm zur Verfügung standen. Als Hauptquellen bezeichnet er zwei handschriftliche Sammelwerke, von denen das eine die in drei Quartbänden zusammengestellten Noctes Thorunenses von Johann Samuel Sammet ${ }^{10}$ und das andere die Handschrift von Wernicke waren. Beide Dokumente seien zu dem Zweck angelegt, „einem künftigen Geschichtsschreiber der Anstalt als Grundlage zu dienen ${ }^{11}$. Das eine befände sich im Besitz der Gymnasialbibliothek, das andere in der Ratsbibliothek. Das 1875 publizierte Verzeichnis der Handschriften und alten Drucke der Thorner Gymnasialbibliothek enthält tatsächlich die Handschrift von Sammet, dagegen fehlt die Schulchronik von Wernicke ${ }^{12}$.

Nach Wernickes Tod übergab sein Sohn Ludwig Wernicke ${ }^{13}$, Bürgermeister von Podgórz, in mehreren Schenkungen gedruck-

9 Lehnerdt, Geschichte des Gymnasiums zu Thorn.

10 Zwischen 1771 und 1803 Lehrer am Thorner Gymnasium (verstorben 1817), vgl. A. Lehnerdt, Das Schüler-Album des Thorner Gymnasiums, „Schulprogramm des Gymnasiums zu Thorn“, (1867), S. 3-25, hier S. 4.

11 Lehnerdt, Geschichte des Gymnasiums zu Thorn, S. 17.

12 Vgl. Curtze, Die Handschriften und seltenen alten Drucke, S. 23.

13 Joseph Ludwig Adolf Wernicke, geboren am 25. September 1831, war 
te und ungedruckte Dokumente aus dem Nachlass seines Vaters der Bibliothek des Thorner Gymnasiums. Darunter befanden sich auch Dokumente zur Schulgeschichte, die teils summarisch, aber in Einzelfällen auch mit Titeln in den Schulprogrammen der Jahre 1874 bis 1878 nachgewiesen werden ${ }^{14}$. Die Schulchronik allerdings fehlt in diesen Verzeichnissen, ebenso jene gedruckten Dokumente, die sich unter den Beilagen der Handschrift finden.

Damit ist zu vermuten, dass Wernicke die Schulchronik samt den von ihm gesammelten Beilagen noch zu seinen Lebzeiten, vielleicht vor seinem Umzug nach Berlin, der Thorner Ratsbibliothek übergeben hat. Diese auf den ersten Blick ungewöhnliche Entscheidung - viel naheliegender wäre eine Übereignung an die Bibliothek seiner ehemaligen Schule gewesen - hängt wahrscheinlich mit seinem im Lebenslauf erwähnten Konflikt zusammen, der ihn zu seiner vorzeitigen Pensionierung veranlasst hatte.

Rektor Lehnerdt gibt in seiner 1868 veröffentlichten Beschreibung der Wernickeschen Schulchronik nur 510 Seiten an, während sich die mit anderer Hand vorgenommene Seitenzählung im heute vorliegenden Exemplar bis auf Seite 588 erstreckt. Bei den lose beigefügten Beilagen befinden sich zudem Dokumente, die bis in das 20. Jahrhundert reichen. Wie und durch wen diese Ergänzungen vorgenommen wurden, ist allerdings ebenso wenig zu klären wie der Ort, an dem dies geschehen ist. Denn die Handschrift wechselte zu einem unbekannten Zeitpunkt sowohl den Besitzer wie den Standort.

Unter den losen Beilagen befindet sich ein von der Erfurter Ärztin Dr. Gertrud Kallinowsky geschriebenes, auf den 31. März

der zweitälteste Sohn von Julius Emil Wernicke, vgl. transkribierten Lebenslauf im Anhang.

14 Vgl. „Schulprogramme des Gymnasiums zu Thorn“, (1874), S. 30; ebenda, (1875), S. 53; ebenda, (1876), S. 33; ebenda, (1877), S. 12; ebenda, (1878), S. 15. 
1939 datiertes, also vor Kriegsbeginn verfasstes und an die Staatsbibliothek Berlin gerichtetes Schreiben, in dem sie mitteilt: „Auf den Wunsch meines vor einigen Monaten verstorbenen Mannes Dr. Georg Kallinowsky - Erfurt übersende ich Ihnen das Paket mit Schriften das Thorner Gymnasium betreffend. Mein Mann ließ bitten diese Schriften so lange in Berlin aufzubewahren bis Thorn wieder Deutsch ist. Mein Mann war in Thorn geboren und hat das dortige Gymnasium besucht".

Bei der Durchsicht der Schuljahresberichte des Thorner Gymnasiums ließ sich allerdings ein Abiturient namens Georg Kallinowsky nicht ermitteln. Stattdessen findet sich im Bericht 1891/1892 ein Fritz Kallinowsky, der Michaelis 1891 sein Abitur ablegte und der ältere Bruder Georgs war. Georg Kallinowsky, geboren am 27. Mai 1871, studierte nach dem Abitur Medizin, promovierte im Februar 1898 (Über Cleidotomie) und heiratete in zweiter Ehe Gertrud, die Absenderin des der Handschrift beigefügten Briefs. Er verstarb am 24. November 1938, also wenige Monate bevor seine Frau die Handschrift an die Staatsbibliothek übersandte.

Die Bedingung, die mit der Bitte um Aufbewahrung verbunden wurde - „bis Thorn wieder Deutsch ist“ - weist auf den Tatbestand hin, dass die ehemals westpreußische Stadt Thorn nach den Versailler Friedensverhandlungen 1920 polnisch wurde. Zwar kam Thorn nach dem Überfall der deutschen Wehrmacht auf Polen ab dem 7. September 1939 wieder unter deutsche Verwaltung und das Thorner Gymnasium wurde noch im gleichen Jahr als Deutsche Oberschule weitergeführt, aber die Handschrift verblieb weiterhin in der Berliner Staatsbibliothek, was angesichts der Zeitumstände leicht zu erklären ist.

Wann die Thorner Schulgeschichte schließlich von der Staatsbibliothek Berlin an ihren heutigen Standort gelangte, ist aufgrund fehlender Übergabedokumente nicht mehr zu ermitteln. Mit hoher Sicherheit geschah dies allerdings lange vor 1989. Ebenso 
wenig lässt sich ermitteln, wie Dr. Georg Kallinowsky in den Besitz der Handschrift gelangte; auch entsprechende Nachfragen bei seinen Kindern ergaben keine weiterführenden Hinweise. Bei den beiden Kindern, die natürlich mittlerweile hochbetagt sind, handelt es sich um einen in Erfurt lebenden pensionierten Pfarrer und eine in Berlin lebende emeritierte Professorin der Freien Universität Berlin. Beide versichern, dass ihr Vater ein überaus korrekter Mann gewesen sei, der nur rechtmäßig in den Besitz der Handschrift gelangt sein könnte.

Georg Kallinowskis Vater war seit den 1890er Jahren bis zu seinem Tod Anfang des 20. Jahrhunderts als Schuldiener im Thorner Gymnasium angestellt. Diese enge Beziehung der Familie Vater Schuldiener, mindestens einer seiner Söhne Schüler - macht es verständlich, dass Georg Kallinowski die Handschrift der Schule erwarb, als sie ihm angeboten wurde. Was ihn schließlich zu der Verfügung veranlasste, die Handschrift nach seinem Tod an die Staatsbibliothek Berlin abzugeben, ist nicht bekannt. Auch seine Kinder konnten darüber keine Auskunft geben, ja, sie wussten nicht einmal, dass dieses Dokument einst im Familienbesitz war.

Wernicke konnte bei der Erarbeitung seiner Schulchronik auf einen reichen Quellenbestand zurückgreifen. Dies zeigt sich sowohl an den von ihm abgeschriebenen als auch in der Beilage gesammelten Dokumenten. Wenn man von vereinzelten Nutzern im 19. Jahrhundert wie etwa Rektor Lehnerdt absieht, handelt es sich um eine in der bildungshistorischen Forschung nahezu unbekannte Quelle. Insofern kann man von einer Wiederentdeckung eines einzigartigen schulgeschichtlichen Dokuments sprechen, das zudem für deutsche wie polnische Bildungshistoriker gleichermaßen von Interesse ist. 


\section{ANHANG}

LEBENSBESCHREIBUNG JULIUS EMIL WERNICKES AUS DER CHRONIK DES THORNER GYMNASIUMS, S. 381-382*

D. 18ten Februar trat als 2ter Unterlehrer ein Dr. Julius Emil Wernicke, geb. zu Breslau d. 16. Aug. 1802. Sein Vater, Friedrich Ludwig Wernicke, war Justizrath und Notarius publicus bei dem König. Oberlandesgericht zu Breslau; die Mutter, Susanne Juliana 2te Tochter des dasigen Bürgers und Seifensieders Hallmann [welche bei ihrem Sohn in Thorn den 2. August 1839 an Altersschwäche starb]. Der Vater [das jüngste von 8 Geschwistern, die alle bereits verstorben] aber starb ihm, da er erst 18 Wochen alt war, d. 26sten Dezbr. 1802, und so blieb er der Erziehung seiner Mutter allein überlassen. Den ersten Elementar-Unterricht, im Lesen, Schreiben und Rechnen, erhielt er in einer dort zu Breslau bestehenden Volksschule, worauf er 91/2 Jahr alt in das Elisabetanum ${ }^{15}$ als Schüler der 5ten Ordnung aufgenommen wurde. In den obern Klassen waren seine vorzüglichsten Lehrer, Etzler Rector der Anstalt, und die Professoren Adolf Muntzel (jetziger Konsistorialrath und Vorsitzender der Prüfungskommission) und Nieckel, unter deren Leitung er dahin kam bereits 1819 d. 19. September [17 Jahre alt] die vorschriftsmäßige AbiturientenPrüfung zu bestehen. Er bezog [Mit dem Zeugniß vollständiger Reife versehen bezog er] hierauf in eben diesem Jahre die Uni-

* BBF/Deutsches Institut für Internationale Pädagogische ForschungArchiv: Thorner Gymnasium, S. 381-382. Der Text wird unverändert wiedergegeben, einschließlich von Eigentümlichkeiten der Orthographie, Kürzungen und Hervorhebungen, spätere Ergänzungen am Blattrand werden in ekkige Klammern gesetzt. Abbrechungszeichen bei Abkürzungen - eine l-ähnliche Schlinge - werden ignoriert.

15 Elisabetanisches Gymnasium. 
versität zu Berlin ${ }^{16}$ um Theologie zu studieren, und wurde unter dem Rectorate Göschens ${ }^{17}$ in die Zahl der akademischen Bürger aufgenommen und den 1sten Novbr. unter Schleiermachers Dekanat in die theologische Fakultät eingeschrieben [zu Berlin immatriculirt d. 27/10 1819]. Später aber gab er den Plan nur Theologie zu studieren auf, und ließ sich unter d. 20. Juni 1820 in die Philologische Fakultät einschreiben, um sich ausschließlich der Philologie zu widmen. Während seiner Studienjahre besuchte er die Vorlesungen Böckh ${ }^{18}$, Wolf [Friedr. August], Hegel ${ }^{19}$, v. Raumer ${ }^{20}$, Ideler ${ }^{21}$, Karl Ritter, Wilken ${ }^{22}, \ldots{ }^{23}$, Neander ${ }^{24}$, Schleiermacher ${ }^{25}$, Osann ${ }^{26}$. Er hatte sich aber in dieser Zeit einer ausgezeichneten Liebe seiner Lehrer und anderer Professoren, unter denen K.G. Zumpt ${ }^{27} \mathrm{zu}$ nennen, wie auch ausgezeichneter Gunst von Seiten des König. Cultus-Ministeriums zu erfreuen, was unter dem Präsidio Altensteins ihn mit nicht unbedeutenden Summen zur Fortsetzung seiner Studien unterstützte. Gegen Ende des Jahres 1821 bestand er vor der wissenschaftlichen PrüfungsCommission zu Berlin sein Examen und wurde 1822 durch das König. Ministerium zum Oberlehrer an das Gymnasium zu Wittenberg bestellt, konnte aber wegen anderweitig eingetretener Hinderniße diese Stelle nicht annehmen. Er kehrte hierauf von

\footnotetext{
16 Die heutige Humboldt-Universität zu Berlin.

17 Johann Friedrich Ludwig Goeschen.

18 August Boeckh.

19 Georg Wilhelm Friedrich Hegel.

20 Friedrich von Raumer.

21 Christian Ludwig Ideler.

22 Friedrich Wilken.

23 Nicht lesbar.

24 Johann August Ludwig Neander.

25 Friedrich Daniel Ernst Schleiermacher.

26 Friedrich Gotthilf Osann.

27 Karl Gottlob Zumpt.
} 
Wittenberg nach seiner Vaterstadt Breslau zurück, wurde daselbst Mitglied des König. Pädagogischen Seminarii und wurde als solches an dem Magdalenum und Fridricianum daselbst mit Lehrstunden beschäftigt. Mittlerweile bereitete er sich vor zur Erlangung der höchsten akademischen Würde, welche er nach Vertheidigung seiner Dissertation, De rebus Cyrenaeorum ${ }^{28}$, unter dem 27sten Februar 1824 erlangte. Ein an ihn ergangener Rufe zur Uebernahme einer Lehrstelle am Gymnasium zu Tilsit ${ }^{29}$ lehnte er ab, wie auch einen anderen an das Gymnasium zu Oels ${ }^{30}$, wogegen er die Berufung nach Thorn annahm; sein hiesiges Lehramt schon den 18. Februar antrat, und den 29. April 1824 am Tage des öffentlichen Schul-Examens eingeführt und vereidigt wurde. Seine Antrittsrede handelte: de incunditate muneris Scholastici [nach ihm hat keiner der eingeführten Lehrer eine lateinische Inaugural-Rede gehalten]. Den 17ten August desselben Jahres verheirathete er sich zu Breslau mit Amalie Friederike Theodora, zweiter Tochter des Kriminal-Raths und Justizkommissarius Friedrich Samuel Küntzel, geb. d. 30. October 1799. Ostern 1828 wurde er von der 2ten Unterlehrerstelle zum 3ten Oberlehrer für das Fach der alten Sprachen befördert [In den Jahren 1834 ff. war er auch Lehrer an der höheren Töchterschule], 1838 rückte er in die zweite Lehrstelle ein, und wurde 1840 durch Erlass des König. Ministerii der geistlichen Angelegenheiten, nachdem er vorher 1839 die erste Lehrstelle erhalten, zum König. Professor ernannt ${ }^{31}$. Häusliche Leiden und Verfolgungen mancherlei Art [und gerade in Thorn recht grell hervortretend], bestimmten ihn schon im Jahre 1845 seine Entlassung nachzusuchen, die ihm Anfang 1846 seit März mit Pension ertheilt wurde.

28 Eine Druckfassung ist nicht zu ermitteln.

29 Heute Sowetsk (Russland).

30 Heute Oleśnica (Polen).

31 Vgl. „Schulprogramm des Gymnasiums zu Thorn“, (1840), S. 14. 
Er lebt seitdem als Privatmann zu Thorn. Aus seiner glücklichen Ehe, die geteilt wurde durch den Tod der Gattin am 14. Febr. 1845 [und von denen noch sechs Lebende an ihrem Grabe weinten und ihren Verlust schmerzlich empfanden] entsproßten 9 Kinder, 4 Söhne und 5 Töchter

1. Ludwig Eduard Emil geb. d. 7ten Januar $1827 \dagger 17$ Jahre 4 Monate alt d. 27. April 1844.

2. Joseph Ludwig Adolf geb. 25. Septbr. $1831 \dagger$

3. Maximilian Julius (...) geb. 23 ten Octbr. $1835 \dagger 29$. Juni 1837

4. Johannes Maximilian Heinrich geb. 24. Juni 1837.

5. Amalie Juliane Franziska geb. 26. August 1825

6. Clara Amalie Pauline Adelyunde geb. 22. Juni $1828 † 26$. Juni 1830

7. Wilhelmine Alexandrine Karoline geb. 22. April 1830.

8. Emilie Wilhelmine Euphemia geb. 17. Septbr. 1833

9. Marie Luise geb. 19. Juli 1840

\section{Schriften}

1. Res Cyrenaeorum 1824 Inauguraldissertation

2. de epigrammatis, quae vulgo Platoni philosopho adscribuntur. Berol $18241 \frac{1}{2}$ Bog. $8^{\circ}$

3. Ueber den griechischen Accent für Schulen bearbeitet. Berlin $^{32} 31 \frac{1}{2}$ Bog. $8^{\circ}$ u. 4 Blätt. Vorrede (recens. Hallsche Literaturztg. Juli 1826 Nro. 174. Allgemeine Schulzeitg. Abth. II Nro. 49.1827)

4. De Elocutione Taciti Programm 1829. $4^{\circ} .4$ 1/2 Bogg.

5. De Elocutione Taciti Specimen primum Thorun 1830. $8^{\circ}$ $7 \frac{1}{2}$ Bog.

6. Allgemeine Andeutungen bei Lesung des Homers. Berlin 1831. $8^{\circ} 11$ Bogen.

32 Erschienen 1825. 
7. Ueber die Bedeutung u. Wichtigkeit der am 27ten Dezember $1831 \mathrm{zu}$ begehenden Festfeier, der vor 600 erfolgten Gründung der Stadt Thorn. 1831. $4^{\circ}$ 3/4 Bogen.

8. Topographisch-historisch-statistische Beschreibung der Stadt Thorn und ihres Gebietes; Vorzeit und Gegenwart umfassend von Dr. Karl Gotthelf Prätorius, nach dem Tode des Verfassers herausgegeben von D.J.E. Wernicke. Thorn 18323 Hefte 27 Bogen.

9. Lineamenta Artis Latine Scribendi exemplis illustrata, Scriptio I quae de Lectione et Excercitatione generatim agit. Thorn 1833 Programm 1 Bog.

10. Sisenniana sive Lucii Cornelii Sisennae Rerum Romanorum Scriptoris Vita et quae supersunt Operum fragmenta. Thorn 1839. $4^{\circ} 7$ Bogg.

11. Geschichte Thorns aus Urkunden, Dokumenten und Handschriften bearbeitet. Thorn 1839. 1842. 2 Bd. $8^{\circ}$ (wofür er begleitet von einem huldvollen Kabinets-schreiben Sr. Majestät des Königs Potsdam d. 30. April 1842 die goldene Huldigungs Medaille des Jahres 1840 erhielt) ${ }^{33}$.

12. Italien im Verhältnis zur Natur und der Geschichte des Menschen. Thorn 1843. $4^{\circ}$. 6 Bogg.

13. Wegweiser durch Thorn und seine Umgebungen mit Abbildungen. Thorn $1846.8^{\circ} 6$ Bogen.

Außerdem Gedichte und verschiedene Aufsätze in den Thornschen Wochenblättern ${ }^{34}$.

33 J.E. Wernicke, Geschichte Thorns aus Urkunden, Dokumenten und Handschriften, 1: Die Jahre 1230-1530 umfassend, 2: Die Jahre 1531-1840 umfassend, (1842).

34 Weitere sechs, kaum lesbare Literaturangaben (14-19) wurden nachträglich am Rand vermerkt. 


\section{ODNALEZIENIE RĘKOPIŚMIENNEJ KRONIKI TORUŃSKIEGO GIMNAZJUM AUTORSTWA JULIUSA EMILA WERNICKEGO}

(STRESZCZENIE)

$\mathrm{W}$ artykule przedstawiono zachowaną w postaci pokaźnego rękopisu kronikę toruńskiego Gimnazjum. Autorem tego, znajdującego się obecnie w zbiorach berlińskiej Bibliothek für Bildungsgeschichtliche Forschung (BBF), dzieła jest Julius Emil Wernicke (1802-po 1858), który od 1824 roku do swojej przedwczesnej emerytury w roku 1846 pracował w Gimnazjum na stanowisku nauczyciela. Wykładał tu grekę i łacinę, przez pewien czas także religię, a od roku szkolnego 1838/1839 dodatkowo również historię i geografię. W 1839 roku J.E. Wernicke awansował na stanowisko przewodniczącego kolegium nauczycielskiego, a rok później nadano mu tytuł profesorski. Prace nad kroniką szkolną prowadził głównie w latach 1840-1847. Jeszcze za swojego życia autor przekazał rękopis toruńskiej Bibliotece Radzieckiej, gdzie - jak udało się wykazać źródłowo - przechowywano go przynajmniej do roku 1868. Nie wiadomo, kiedy dokładnie kronika znalazła się w posiadaniu Georga Kallinowskiego, który koło 1890 roku był uczniem toruńskiego Gimnazjum. Na początku 1939 roku, już po jego śmierci, żona przekazała rękopis Bibliotece Państwowej w Berlinie, skąd później trafił do BBF.

Najobszerniejszą część kroniki jej autor poświęcił chronologicznemu omówieniu ważnych dla Gimnazjum wydarzeń, oczywiście począwszy od momentu założenia tej uczelni. Nieduże fragmenty poświęcone zostały innym toruńskim szkołom: św. Jana, św. Jakuba, szkole nowomiejskiej, a także Kolegium Jezuickiemu. Dużą część pracy stanowi rozdział dotyczący organizacji i życia gimnazjum (s. 66-436), w którym przedstawieni zostali poszczególni rektorzy i nauczyciele (mowa jest tu o ich wykształceniu, sytuacji rodzinnej i publikacjach). 
Opracowując kronikę toruńskiego Gimnazjum J.E. Wernicke korzystał z bogatych zbiorów archiwalnych, o czym świadczą zarówno sporządzone przez niego kopie dokumentów, jak i załączone oryginalne fragmenty dokumentacji. Jedynie w XIX wieku odnotowano pojedyncze przypadki korzystania $\mathrm{z}$ kroniki, poza tym stanowi ona niemalże całkowicie nieznany materiał źródłowy. Można więc mówić o ponownym odkryciu tego niezwykłego dokumentu, interesującego w równym stopniu dla polskich i niemieckich historyków.

Tłumaczenie/Übersetzt von Barbara Sapała 